\title{
Variations
}

Variations

Revue internationale de théorie critique

16 | 2012

Tahrir is here !

\section{Le mode artistique de la Révolution : de la gentrification à l'occupation (I)}

\section{Martha Rosler}

Traducteur : Lucia Sagradini

\section{(2) OpenEdition}

\section{Journals}

Édition électronique

URL : http://journals.openedition.org/variations/206

DOI : 10.4000/variations.206

ISSN : 1968-3960

\section{Éditeur}

Les amis de Variations

Édition imprimée

Date de publication : 16 janvier 2012

Référence électronique

Martha Rosler, «Le mode artistique de la Révolution : de la gentrification à l'occupation (I) », Variations [En ligne], 16 | 2012, mis en ligne le 20 février 2012, consulté le 30 avril 2019. URL : http://

journals.openedition.org/variations/206 ; DOI : 10.4000/variations.206

Ce document a été généré automatiquement le 30 avril 2019.

Les ami•e•s de Variations 


\title{
Le mode artistique de la Révolution : de la gentrification à l'occupation (I)
}

\author{
Martha Rosler \\ Traduction : Lucia Sagradini
}

\section{NOTE DE L'ÉDITEUR}

Première partie de l'article « The Artistic Mode of Revolution: From Gentrification to Occupation ». La seconde partie sera publiée dans Variations $n^{\circ} 17$.

Première publication sur www.theoriecritique.com, « Tahrir is here ! », 16 janvier 2012, pp. 49-55

1 L'événement crucial que constituent les démonstrations et occupations publiques des dernières semaines, mois, et même de l'année passée, coiffent toute la discussion sur les luttes, les exodes et les réappropriations du travail cognitif et créatif, en particulier dans le champ des arts visuels. J'ai choisi d'aborder les thèses au sujet de la « classe créative » à travers ce détour, afin d'élaborer mes arguments à la lumière de ces occupations, de ces révoltes, dans le dessein de réaliser quelques observations sur la relation entre les artistes, la prise de position de la classe créative et le mouvement oppositionnel.

2 Avant même que la "multitude» devienne une référence courante pour rêver la révolution, il y a eu le célèbre moment Seattle en 1999, lorsque les protestations altermondialistes ont fait converger les écologistes et la communauté des activistes avec les organisations syndicales pour bloquer un meeting de l'Organisation mondiale du commerce, un scénario qui s'est répété par la suite dans de nombreux endroits et dans plusieurs pays. Ce n'est pas nouveau, mais les processus réunis sous le nom de mondialisation, et qui se concentrent dans les flux du capital, des biens et du travail, créent une unité qui ne sert pas toujours l'intérêt dudit capital ou des capitalistes.

3 Nouriel Roubini, explorant Marx, a écrit dans L'instabilité de l'inégalité que «le capitalisme non réglementé peut conduire à des épisodes réguliers de surcapacité, de sous-consommation, et provoque la récurrence de crises financières destructrices, 
alimentées par des bulles de crédit et des prix des actifs ainsi que des faillites $»^{1}$. Roubini nous signale que le capitalisme tend à des effondrements catastrophiques - rien de neuf ici diriez-vous. Cependant, le point important est que le néo-libéralisme et ses financiarisations rampantes se présentent désormais à nous comme un capitalisme qui dévore ses propres enfants. Roubini rappelle à ses lecteurs (ou explique à ses lecteurs nord-américains qui n'en ont aucune idée) que déjà avant la Grande Dépression, la bourgeoisie éclairée s'est rendue compte que le système de protection sociale et de redistribution qui s'engage dans le bien public -éducation, santé, et sécurité sociale- était indispensable pour empêcher la révolution ${ }^{2}$.

4 Roubini remarque, de plus, que l'État providence moderne a surgi à travers la stabilisation macro-économique nécessaire pour sortir de la grande dépression, qui exigeait « le maintien d'une classe moyenne importante, l'élargissement de disposition de biens publics par le biais de taxations progressives, tout en favorisant les opportunités économiques pour tous ». Mais tout cela a disparu sous la politique massive de déréglementation des années Reagan-Thatcher, que, Roubini, pas marxiste du tout, rattache «aux manquements du modèle de protection sociale européen... qui se manifestent dans des déficits budgétaires béants, la surréglementation étatique, et une perte de dynamisme économique $»^{3}$.

5 Roubini, contrairement à beaucoup, proclame pourtant l'échec du modèle «angloaméricain ", et précisément du modèle financier qui a saisi l'imagination et conduit la politique des élites au pouvoir de l'ancien Bloc de l'Est, dont nombreux sont en train de détruire les classes moyennes actuelles et futures (pensez à la Lettonie) selon un modèle du néo-thatchérisme de la Grande-Bretagne ${ }^{4}$. Citibank, par exemple, a nécessité deux sauvetages du gouvernement américain après la crise financière de 2008, ce qui seul lui permet d'afficher des profits trimestriels record de 3,8 milliards de dollars US, une augmentation de $74 \%$ par rapport au trimestre précédent. Son chef, Vikram Pandit, a ensuite exprimé sa sympathie avec les manifestants d'Occupy Wall Street, qu'il s'est proposé de rencontrer.

6 L'impulsion des révoltes dans le monde entier a trouvé son inspiration dans le printemps arabe qui a traversé le Maghreb, poussé par la frustration des jeunes des classes moyennes éduquées, qui ont peu d'espoir en un avenir meilleur pour eux-mêmes, malgré leurs études universitaires ce qui est relativement nouveau, ce qui les pousse à la confrontation à des sociétés contrôlées par des élites dirigeantes extrêmement puissantes. Ce sont des sociétés qui n'ont fait aucun effort pour créer l'État providence moderne ou même des États à la gestion néolibérale, susceptible de contrôler la corruption, de limiter le cynisme bureaucratique et au népotisme flagrant, et qui n'ont pas été en mesure d'instituer des États ayant l'apparence d'une gouvernance démocratique. D'ailleurs, les opposants dans le monde occidental sont assez conscients que les conditions politiques de leur action sont assez proches partout dans le monde, si on les considère de manière fonctionnelle et pratique ${ }^{5}$.

7 Pareilles protestations, comme en France en 2006 avec la mobilisation générale contre la "précarisation », ont un lien avec la colère des jeunes des classes populaires qui ont alors convergé avec les jeunes de la classe moyenne, ou alors laissent éclater leur rage contre les violences policières racistes, dans les soulèvements antérieurs des banlieues en 2005 ou, il y a quelques mois en Angleterre, lorsque les banlieusards ont détruit et pillé aux côtés de jeunes de la classe moyenne. À Londres, certains de ces derniers groupes s'étaient déjà mobilisés un mois plus tôt, comme les jeunes Chiliens, en grande partie à 
cause du coût de la scolarité qui les broie. Les manifestations de ces groupes, de ces classes, sont parties par le constat commun qu'il n'y aura probablement pas de sécurité de l'emploi pour personne, ou même pas d'emploi du tout. Ainsi, la précarisation ne découle pas nécessairement d'une forme particulière de travail.

La précarisation rejoint un ensemble de stratégies pour rétablir la rentabilité après la crise de l'après-guerre. L'automatisation: le remplacement des travailleurs par des machines ; la délocalisation : la quête mondiale du capital d'aligner droits des travailleurs et réglementation environnementales vers le bas; et la financiarisation : le maintien de la valeur excédentaire sur le marché boursier, par opposition à la plus-value extraite de la production. Ces stratégies complètent les assauts plus largement observés à l'encontre de l'État providence et contre les droits des travailleurs ${ }^{6}$.

Beaucoup d'étudiants et de jeunes diplômés qui protestent ont été préparés pour les emplois dits des industries de la connaissance, plus récemment désignées comme étant les industries créatives. Permettez-moi de prendre un peu de recul ici, et de rappeler la consolidation de ce secteur dans l'ère de l'information naissant, au début des années 1960. Clark Kerr, économiste du travail, premier chancelier de l'Université de Californie (Berkeley, campus d'élite), puis président de l'ensemble du système de communications unifiées, pense l'université comme un site de production de travailleurs de la connaissance. En 1960, il a supervisé la création d'un plan directeur pour une croissance agressive au XXIe siècle, alliant les établissements publics de l'enseignement supérieur en trois niveaux : les universités de recherche, les « collèges d'État » et la formation de deux ans de "junior colleges", rebaptisés "collèges communautaires", décernant des diplômes. Ce plan de compétition internationale implique d'unifier la formation et l'administration du secteur de la connaissance tout entier, en y intégrant les élites de tous les domaines de base, mais également les classes laborieuses "post-fordistes ", qu'elles soient industrielles, médicales, juridiques ou financières, et ce dans un monde politiquement divisé. Kerr a pointé l'université comme un «instrument privilégié de la stratégie nationale » et il a considéré que "l'industrie de la connaissance » (se sont ses termes) allait supplanter les industries entourant les nouveaux modes de transports ferroviaires au XIXe siècle et les automobiles du XXe - dans l'unification de la nation, agissant en tant que figure de proue économique, et servant de moteur à la domination américaine.

Le mouvement étudiant fondateur des années 1960, le Mouvement Free Speech de Berkeley, était une réaction contestataire véhémente aux politiques éducatives et managériales de Kerr ainsi que de ses objectifs. Il a ainsi été un mouvement constitué de membres de la classe moyenne, destinés à devenir les travailleurs d'élite de l'industrie des nouvelles connaissances, ou même les élites dirigeantes. Force est de constater que le système universitaire Californien est aujourd'hui au bord de la rupture, comme le dictionnaire interne d'Apple nous en informe dans sa définition du mot baromètre : « les campus sont souvent le baromètre du changement ».

11 À titre de comparaison, la sous-culture punk des années 1970 en Grande-Bretagne était sans doute une réponse des classes laborieuses à un avenir décati, en dépit des marques qu'il a en partie imprimées dans les écoles d'art, qui étaient alors un nouvel espace expérimental pour les classes laborieuses inadaptées. Comme Dick Hebdige le décrit :

Malgré l'assurance hardie des politiciens tant travaillistes que conservateurs dans l'idée que « nous n'avons jamais vécu de manière aussi aisée », les classes sociales ont refusé de disparaître. La façon dont les classes sociales ont été vécues, les formes dans lesquelles 
l'expérience de classe a trouvé son expression dans la culture, a pourtant changé de façon spectaculaire. L'avènement des médias de masse, les changements dans la composition de la famille, dans l'organisation de l'école ou du salariat, les changements dans le statut relatif du travail et des loisirs : le tout servit à fragmenter et à polariser la communauté des classes laborieuses, en produisant une série de discours marginaux dans le cadre des expériences de classe ${ }^{7}$.

En ce sens, le Punk était viscéralement une expérience anti-marchandise et anticorporatiste, et il a suivi une tactique d'enlaidissement et d'automutilation, dans un grand « fuck you » à la culture bourgeoise ; le fait qu'il ait été rapidement transformé en marchandise et fortement encouragé par l'industrie de la musique n'était pas le problème... Au moins, jusqu'à ce qu'il ne le devienne. Car la vie politique est devenue presque indistincte de la vie quotidienne pour les générations après les années 1970, et ce cadre de références s'est désormais propagé à travers le monde.

Le mode de vie a, en parallèle, été intensivement développé comme axe majeur pour la commercialisation des biens de consommation, encadré par des tableaux complexes de profils de consommateurs, afin de réjouir le cœur de toute agence de publicité et d'impressionner n'importe quel client. On trouve des pépites dans l'analyse marketing du mode de vie réalisé par Theodore Levitt, professeur à Harvard de Business Administration, et du Marketing, quand, autour de 1984, lorsqu'il a commenté l'échec de la société Hoover à vendre des machines à laver en Europe : « Ils ont demandé aux gens quelles fonctionnalités ils voulaient pour une machine à laver plutôt que ce qu'ils voulaient pour profiter de la vie $»^{8}$. Levitt, responsable de la Harvard Business Review, est aussi celui à qui l'on doit la popularisation du terme de «mondialisation». Dans The Marketing Imagination, son best-seller de 1983, Levitt a éclairé la marchandise avec ce qu'on appelle "high-touch", biens d'un grand attrait -jeans et Coca-Cola- en correspondance avec la haute technologie, ces biens qui sont au cœur de la dynamique commerciale des États-Unis pour vendre ses produits dans le monde.

Il nous faut constater qu'une puissante force entraîne le monde vers une communauté convergente, et cette force est la technologie. Presque tout le monde veut partout dans le monde toutes les choses dont ils ont entendu parler, vu ou expérimenté via les nouvelles technologies.

se réfère à l'homogénéisation comme le moyen et le résultat de la mondialisation. I différencie les multinationales des sociétés mondiales, qui, dit-il , « vendent des produits standardisés de la même manière partout - automobile, sidérurgie, chimie, pétrole, ciment, produits agricoles et équipements, industrie et commerce, services bancaires et assurances, ordinateurs, transports, instruments électroniques, pharmaceutiques et télécommunications ", pour n'en citer quelques-unes des plus évidentes.

Trente ans plus tard, nous voudrions regrouper plusieurs de ces catégories sous la rubrique des industries de la connaissance, incluant la gestion de la production industrielle fordiste. Depuis trente ans, les politiques des styles de vie, qui rassemblent et différencient à la fois, décident comment nous vivons ou comment nous sommes censés vivre. Les gens forment des alliances et des identités basées sur des affinités de goût, formés par-dessus tout par le tribalisme de l'apparence. Ainsi marchandisés, les modes de vie comprennent non seulement des objets, mais des personnes, réalisations et enfants, et ils ont tendance à être coûteux tant à réaliser qu'à entretenir. Le Punk est désormais un choix de vie comme un autre, certes romantique et urbain. Les punks, les gothiques, entre autres, associés au East Village de New York fournissent l'uniforme préféré des 
mécontents des banlieues et des petites villes, tout comme le Bronxish hip hop, mondialement populaire, fournit un style de vie pour les classes laborieuses d'origine immigrée.

Dans cette taxonomie, l'hipsterism des branchés est le mode de vie arty - le triomphe de la surface sur le fond - qui est une conséquence directe de la disponibilité des biens culturels par des moyens technologiques.

Pendant ce temps, artistes et groupes d'artistes autogérés entre autres, se trouvent au centre d'une classe créative bohème, qui a instauré, dynamisé et élaboré stratégiquement le mouvement Occupy Wall Street à New York qui a eu lieu au Zucotti Parc, désormais rebaptisé Parc de la Libertés.

\section{NOTES}

1. http://www.economonitor.com/nouriel/2011/10/14/from-project-syndicate-the-instabilityof-inequality/ and http://www.economonitor.com/nouriel/2011/10/17/full-analysis-theinstability-of-inequality/. Roubini a commencé ce blog le 11 octobre 2011 en signalant les «troubles sociaux et politiques ainsi que l'instabilité à travers le monde avec les masses de gens dans les rues réelles et virtuelles»: «le printemps arabe; les émeutes à Londres; les manifestations en Israël des classes moyennes; celles des étudiants chiliens, la destruction en Allemagne des voitures chères des « gros bonnets »; le mouvement en Inde contre la corruption; les tragédies liées à la corruption et aux inégalités en Chine; et maintenant «Occupy Wall Street », mouvement qui va de New York et traverse les Etats-Unis »

2. J'ai abordé cette question dans mon essai, In, Around and Afterthoughts: On Documentary Photography, (1981) sur le rôle de la photographie documentaire dans l'organisation des images idéologiques, afin de mobiliser des soutiens pour les très pauvres. Ce raisonnement était marqué par l'esprit de l'administration Roosevelt.

3. Roubini, http://www.economonitor.com/nouriel/2011/10/14/from-project-syndicate-theinstability-of-inequality/. Je choisis de me référer à Roubini, mais j'aurais très bien pu choisir un autre parmi les quelques autres économistes, comme Joseph Stiglitz et Paul Krugman du New York Times, pour pointer les peurs de la gauche libérale des économistes occidentaux.

4. La Commission européenne a récemment voté une série de mesures qui conditionnent les capacités des États membres à contrôler leurs budgets, rétablit la limite de $3 \%$ du Traité de Maastricht sur les déficits et les dettes de $60 \% \mathrm{du}$ PIB, au-delà duquel des amendes importantes seront imposées, entre autres mesures. Selon l'économiste Susan George, la Commission est également le maître d'œuvre d'un changement dans la protection des travailleurs menant à des semaines de travail plus longues, des salaires inférieurs, et plus tard touchant aux retraites. Susan George, "A Coup in the European Union?» CounterPunch (14 oct. 2011), http:// www.counterpunch.org/2011/10/14/a-coup-in-the-european-union/.

5. Les réponses d'Europe Occidentale sur les perspectives d'avenir, tels que le campement des Indignados en Espagne et les manifestations en Grèce place Syntagma sont des exemples essentiels, le soulèvement en Tunisie a finalement été un succès, aux vues de l'ampleur et de la faible probabilité de succès de l'occupation de la place Tahrir du Caire, et il a été la pierre de touche du mouvement. Les occupants vétérans de la place Tahrir ont d'ailleurs envoyé un 
message à Occupy Wall Street: «La crise actuelle en Amérique et en Europe occidentale a commencé à pénétrer votre vie à vous aussi, c'est choses dont nous avons fait l'expérience, le dos cassé par l'endettement personnel et l'austérité publique. Non content d'anéantir les restes de la sphère publique et de l'État Providence, le capitalisme et l'austérité étatique aujourd'hui attaquent même la sphère privée et le droit des personnes à un travail décent, ainsi des milliers de gens se trouvent à la fois sans toit et endettés auprès des banques qui les ont forcés à tout perdre et vivre dans la rue.

Nous sommes avec vous, pas seulement dans vos tentatives pour faire tomber le vieux monde, mais aussi pour faire l'expérience du nouveau. Nous ne sommes pas là pour protester. Qui est là pour protester? Que pourrions-nous leur demander pour qu'ils nous l'accordent? Nous sommes l'occupation. Nous sommes là pour nous accaparer les espaces de pratiques publiques, y compris ceux qui ont été marchandisés, privatisés et verrouillés dans les mains de la bureaucratie sans visage, de portefeuilles immobiliers et sous la "protection» de la police. Accrochez-vous à ces espaces, nourrissez-les et laissez les croître. Après tout, qui a construit ces parcs, ces places, ces immeubles? Quel travail les rend réel et agréable?

Pourquoi devrait-il être si naturel qu'ils doivent être privés, policés et disciplinés? Reconquérir les espaces et les gérer de manière juste et collectivement est une preuve suffisante de notre légitimité. »

6. Christian Marazzi, The Violence of Financial Capital, trans. Kristina Lebedeva and James Francis McGimsey, Los Angeles, Semiotexte, 2011.

7. Dick Hebdige, Subculture: The Meaning of Style, Methuen, 1979, p. 78.

8. Theodore Levitt, «The Globalization of Markets », The McKinsey Quarterly, Summer 1984, p. 13. «Le cas Hoover illustre bien comment les pratiques perverses du marketing et l'absence de toute imagination dans ce champ entraînent le maintien d'attitudes défavorables des multinationales alors que les clients veulent jouir des bénéfices de la standardisation mondiale. L'ensemble du projet est parti du mauvais pied. Ils ont demandé aux gens quelles fonctionnalités ils voulaient pour une machine à laver plutôt que ce qu'ils voulaient pour profiter de la vie. Lançant ainsi une ligne de produits conçus de façon individualisée pour chaque nation, mais de manière irréfléchie. Les managers, qui étaient fiers de leur pratique de marketing approfondie, n'ont pas en réalité réfléchi du tout. Hoover pose les mauvaises questions, puis les réponses sont appliquées sans réflexion ni imagination $»$.

9. L'occupation de Wall Street a été impulsée par un certain nombre d'événements dont je ne peux que partiellement esquisser ici les grands traits. L'occupation avait été pour ainsi dire annoncée quelques mois plus tôt par Bloombergville, un campement de trois semaines réalisé par des dirigeants syndicaux et des militants, qui s'est tenu au City Hall Park contre les coupes budgétaires draconiennes. Un autre précédent important a été la semaine d'occupation du Wisconsin State House à Madison, soutenu par les syndicats, et même les syndicats de policiers. Un article sur la possibilité d'étendre la contestation de la place Tahrir a été réalisé par l'anarchiste et anthropologue David Graeber, il a été publié par Adbusters, revue canadienne très brillante d'inspiration situationniste. Adbusters a ensuite lancé un appel pour occuper Wall Street le 17 Septembre 2011. Les discussions sur la possibilité de construire un mouvement avaient eu lieu au cours de l'été au 16Beaver, un espace de discussion géré par des artistes dans la zone de Wall Street. Une réunion ad hoc au 16Beaver, après des débats avec des militants et des universitaires au cours de laquelle Graeber a discuté de son travail sur la dette (dette: Les Premières Années 5000, New York, Melville House, 2011). Cette rencontre a donné l'impulsion finale. Le groupe Bloombergville rassemble les contestataires le 17 septembre pour occuper, mais Graeber, en collaboration avec l'artiste et militante anarchiste, Georgia Sagri, qu'il avait rencontré lors du séminaire 16Beaver et le japonais anarchiste activiste de Sabu Kohso, ont organisé l'Assemblée générale sur une ligne anarchiste.

En Octobre 2011, Adbusters a proposé quelques conseils tactiques supplémentaires, plus 
artistiques que militants old-school, mais rodé à la lutte depuis les manifestations de Seattle contre l'OMC en 1999 : «Il est maintenant temps d'aller vers les débordements ... tours déviants, performances subversives et détournements ludiques de tout genre. Laissez aller votre imagination insurrectionnelle. Tout doit connaître une transformation, de l'économie mondiale à l'évolution de la façon dont nous mangeons, la manière dont nous nous déplaçons, de notre façon de vivre, d'aimer et de communiquer ... Nous devons être l'étincelle qui subit une révolution globale de la vie quotidienne!». D'ailleurs, le champ des études de la performance du Département de la New York University accueille actuellement une série hebdomadaire de conférences et d'ateliers axés sur le changement social à travers «les tactiques et les stratégies créatives ».

\section{AUTEURS}

\section{MARTHA ROSLER}

Martha Rosler, artiste new-yorkaise et iconoclaste mondialement connue, est aussi l'auteure d'essais qui s'inspirent de la Théorie critique, nourris de ses rencontres avec Herbert Marcuse ou Frederic Jameson. 